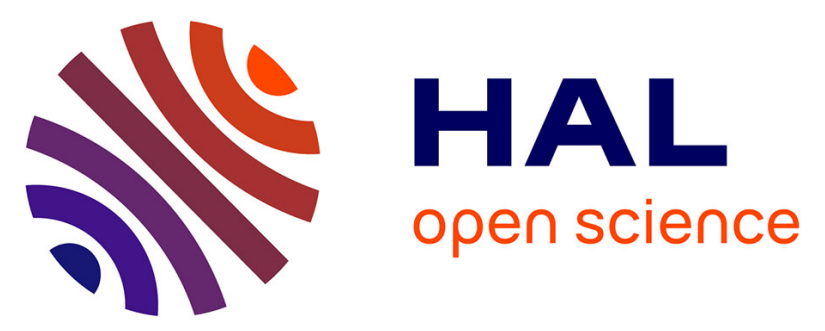

\title{
Modeling Deformable Bodies Using Discrete Systems with Centroid-Based Propagating Interaction: Fracture and Crack Evolution
}

Alessandro Della Corte, Antonio Battista, Francesco Dell'Isola, Ivan Giorgio

\section{To cite this version:}

Alessandro Della Corte, Antonio Battista, Francesco Dell'Isola, Ivan Giorgio. Modeling Deformable Bodies Using Discrete Systems with Centroid-Based Propagating Interaction: Fracture and Crack Evolution. Mathematical Modelling in Solid Mechanics, 2017, Volume 69 of the series Advanced Structured Materials. hal-01511528

\author{
HAL Id: hal-01511528 \\ https://hal.science/hal-01511528
}

Submitted on 21 Apr 2017

HAL is a multi-disciplinary open access archive for the deposit and dissemination of scientific research documents, whether they are published or not. The documents may come from teaching and research institutions in France or abroad, or from public or private research centers.
L'archive ouverte pluridisciplinaire HAL, est destinée au dépôt et à la diffusion de documents scientifiques de niveau recherche, publiés ou non, émanant des établissements d'enseignement et de recherche français ou étrangers, des laboratoires publics ou privés. 


\title{
Modeling deformable bodies using discrete systems with centroid-based propagating interaction: fracture and crack evolution
}

\author{
Alessandro Della Corte, Antonio Battista, Ivan Giorgio and Francesco dell'Isola
}

\begin{abstract}
In this paper we use a simple discrete system in order to model deformation and fracture within the same theoretical and numerical framework. The model displays a rich behavior, accounting for different fracture phenomena, and in particular for crack formation and growth. A comparison with standard Finite Element simulations and with the basic Griffith theory of fracture is provided. Moreover, an 'almost steady' state, i.e. a long apparent equilibrium followed by an abrupt crack growth, is obtained by suitably parameterizing the system. The model can be easily generalized to higher order interactions corresponding, in the homogenized limit, to higher gradient continuum theories.
\end{abstract}

\section{Introduction}

\subsection{Motivation and basic ideas}

Modeling fracture in an effective way has always been a major challenge for solid mechanics. Many sophisticated theoretical and numerical tools have been devel-

Alessandro Della Corte

Department of Mechanical and Aerospace Engineering, Università di Roma La Sapienza e-mail: alessandro.dellacorte@uniroma1.it

Antonio Battista

Laboratory of Science for Environmental Engineering, Université de la Rochelle e-mail: antonio.battista@univ-lr.fr

Ivan Giorgio

MeMoCS, International Research Center for the Mathematics \& Mechanics of Complex Systems, Università dell' Aquila e-mail: ivan.giorgio@uniroma1.it

Francesco dell'Isola

MeMoCS, International Research Center for the Mathematics \& Mechanics of Complex Systems, Università dell'Aquila e-mail: francesco.dellisola@uniroma1.it 
oped, and considerable progresses have been obtained in recent years in the framework of both classical and generalized continuum models (see [1, 2, 3, 4, 5]). However, the richness of the phenomenology is leading to theoretical formulations that are becoming increasingly complex and may be computationally expensive and/or involve a stochastic approach in order to capture the peculiar characteristics displayed by real fractures. The idea behind the present work is to explore a very simple discrete system characterized by a centroid-based propagating interaction, and evolving through actual configurations connected by virtual (i.e., not visible) ones; the system, in its basic version and without including fracture, was introduced in [6], while some preliminary results on fracture have been shown in [7]. The model presents some similarities with Molecular Dynamics (MD) but, as it will be shown, its specific features cannot be recovered within standard MD models.

The potential advantages of the proposed approach are mainly linked to the simplicity of the model. First of all this results in a significantly low computational cost; moreover, deformation and fracture are covered here within the same simple model; finally as we will see, more general continuum theories can be easily numerically investigated by means of slight modifications of the algorithm. Since generalized continua are one of the most promising and rapidly evolving areas in modern mechanics, this last feature of the proposed model may be particularly appealing (for a theoretical coverage on higher gradient theories, see e.g.: $[8,9,10,11]$, and specifically for an approach combining higher gradient theories with lattice models, see [12]). A closely related topic is that of micromorphic/microstructured continua (see $[13,14,15,16]$ for classical references and $[17,18,19,20,21,22]$ for interesting applications), which can be viewed as a generalization of higher gradient theories and may benefit as well from the development of new discrete approaches. What is making these subjects critical, in the opinion of the authors, is the advancements in manufacturing possibilities in the last years, as 3D printing and other computeraided manufacturing techniques, which are resulting in new metamaterials requiring a suitable theoretical description (and related numerical techniques) for objects whose richness at the micro-scale cannot be captured by classical continuum models (see e.g.: [23] for a review of recent results).

Discrete systems are, of course, very frequently studied in order to address the aforementioned problems. In particular, Molecular Dynamics (MD) is by now a very large research area with specific methods and very sound results (see e.g.:[24, 25, 26, 27]). The model presented herein, while sharing certain basic features with MD ones, is characterized by some relevant differences in the approach. $\mathrm{MD}$ is indeed based on the numerical study of systems constituted by a very large number $N$ of elements. The numerical computation of the trajectories of particles in the ordinary $6 N$-dimensional phase space of positions and momenta employs the classical mechanics laws of motion (for a sample of the numerical method the reader can see: $[28,29,30])$. The system investigated in the present paper, on the other hand, does not consider any explicit equation of motion. Instead, in order to simplify the model to the maximum, the elements of our discrete system move according to an interaction law which:

- is purely geometrically based; 
- propagates along square frames centered in special elements (the 'leaders' of the system);

- drives the elements through virtual configurations which are needed for the computation of the evolution, but are not visible in the final output.

An important class of discrete systems for continuous deformable bodies, and in fact a particular case of MD models, is given by masses-springs or beads-springs models, which can be characterized in different ways by changing the geometry of the springs and their type (extensional, torsional). The mass-spring systems are common tools in Computer Graphics for the simulation of soft bodies[31, 32]. There are several relevant differences between these systems and the proposed model. The two probably most important ones are the following:

i. In case of mass-spring system, a uniform motion of the leaders results in a global motion $r(t)=r^{*}(t)+r^{* *}(t)$, with $r^{* *}(t)$ being a periodic function and $r^{*}(t)$ a transient term such that $\left\|r^{*}(t)\right\| \rightarrow 0$ as $t \rightarrow \infty$ [33]. As we will see, no such decomposition makes sense in case of the proposed system. For instance, it can be proven that a uniform motion imposed to a leader results, asymptotically, in a rigid motion of the system.

ii. It can also be proven that uniformly accelerated motion of a set of leaders results in our case in a disaggregation of the system (i.e. there is no $R \in \mathbb{R}$ such that for every $t \in\left[0, \infty\left[\right.\right.$ the system is contained in $\mathscr{B}_{R}(x, y)$ for some $\left.x, y \in \mathbb{R}^{2}\right)$.

The characteristics of our model have, as we will see, several advantages, but at the same time make harder a standard variational formulation. As we will show, energy-based investigations are possible in the discrete context here considered, but the identification of an explicit Lagrangian whose minimization leads to the exact dynamics displayed by the discrete system is far from trivial, and will be one of the main objectives of future investigations. One of the main checks we will perform will concern the systematyc comparison with Finite Element simulations. Nowadays FE analysis is indeed probably the most reliable tool for the numerical simulation of the behavior of deformable bodies, also thanks to the possibility of adapting it to the features of the problem studied. Isogeometric analysis (see for instance $[34,35$, $36,37]$ ), in particular, can be especially convenient for shape optimization problems that easily arise in the study of multi-agent systems moving in unbounded domains and starting form arbitrary configurations, as is the case in our context.

\subsection{A summary of the algorithm and of the formalism employed}

In this section, we will briefly describe the model studied in the present paper. The algorithm and the formalism will be summarized in their most relevant features; the reader can find a fully detailed description in [6].

Let us consider a discrete system $\mathfrak{S}$ constituted by a finite number of points which are characterized by their position in the real plane; we will call them the 'elements' of $\mathfrak{S}$. The elements are set, in the initial configuration $C^{0}$, in the nodes of a squared 
grid sized $L \times L$. We consider a set of discrete time steps $\mathfrak{T}_{\mathfrak{m}}=\left\{0, t_{1}, \ldots, t_{m}, \ldots\right\}$ and an orthonormal reference system with axes parallel to the sides of the system, the unit length being equal to the cell side in $C^{0}$. Each Lagrangian element $(i, j)$ of $\mathfrak{S}$, placed in $\left(x_{i j}^{1}, x_{i j}^{2}\right)\left(t_{m}\right)$, has a set of neighbors

$$
N_{n}(\bar{i}, \bar{j}):=\left\{(i, j) \in C^{0}: \rho[(\bar{i}, \bar{j}),(i, j)]=n\right\}
$$

where $\rho$ is the $\mathbb{R}^{2}$-Chebyshev distance, i.e. the distance in $\mathbb{R}^{2}$ given by $\rho\left(\left(x^{1}, x^{2}\right),\left(y^{1}, y^{2}\right)\right)=$ $\max \left\{\left|x^{1}-y^{1}\right|,\left|x^{2}-y^{2}\right|\right\}$. With this definition, selecting $n=1$ and $n=2$, we have respectively the first and second neighbors as shown in Fig. 1.



Fig. 1 Graphical representation of neighbors: the first and second neighbors of the red element are the ones respectively in yellow and green.

Let us select a leader element $\mathfrak{L}$ whose actual position is defined by a prescribed motion $\mathscr{M}: t_{m} \in \mathfrak{T}_{\mathfrak{m}} \longrightarrow\left(x_{\bar{i} \bar{j}}^{1}, x_{\bar{i} \bar{j}}^{2}\right)\left(t_{m}\right) \in \mathbb{R}^{2}$. We are now ready to describe the interactions between the elements. We will consider virtual steps in between two actual states, i.e. states that are invisible in the real displacements of the system but are necessary for computing its evolution. Let us consider a configuration $V_{0}^{t_{1}}$ such that the leader $\mathfrak{L}$ is positioned in $\mathscr{M}\left(t_{1}\right)$ : this is defined as the first virtual configuration. Then, recalling that by centroid of a set of points $P_{1}\left(x_{1}^{1}, \ldots, x_{n}^{1}\right), \ldots, P_{m}\left(x_{1}^{m}, \ldots, x_{n}^{m}\right) \in$ $\mathbb{R}^{n}$ one means the point $P\left(\sum_{i=1}^{m} \frac{x_{1}^{i}}{m}, \sum_{i=1}^{n} \frac{x_{2}^{i}}{m}, \ldots \sum_{i=1}^{n} \frac{x_{n}^{i}}{m}\right)$, the next virtual configuration $V_{1}^{t_{1}}$ will be defined as the on in which:

1. the leader $\mathfrak{L}$ remains in $V_{0}^{t_{1}}$;

2. every one of its first neighbors $N_{1}(\bar{i}, \bar{j})$ moves to the centroid of its own first neighbors in $V_{0}^{t_{1}}$

3. all others elements remain in the position they had in $V_{0}^{t_{1}}$.

Iterating and generalizing to the $n$-th virtual step, $V_{n}^{t_{1}}$ will be the virtual configuration in which: 
1. the leader $\mathfrak{L}$ together with its first $n-1$-th neighbors are in the same position they had in $V_{(n-1)}^{t_{1}}$;

2. every one of the $n$-th neighbors of $\mathfrak{L}$ has moved to the centroid of its own first neighbors in $V_{(n-1)}^{t_{1}}$;

3. all others elements are in the same position they had in $V_{(n-1)}^{t_{1}}$.

We will get the actual configuration $C^{t_{1}}$ when $n$ equals the maximum Lagrangian Chebyshev distance of the elements from the leader (see Fig. 2).
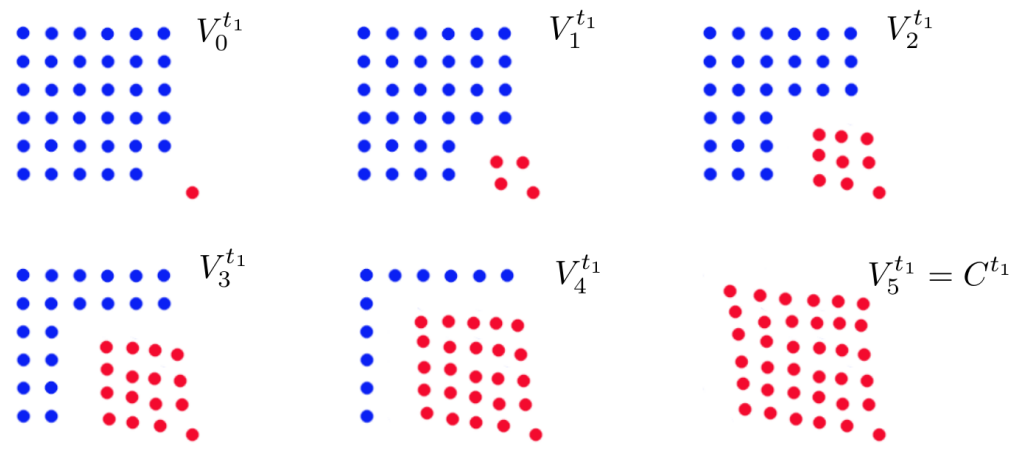

Fig. 2 Graphical representation of the virtual configurations. In red the elements which are already in the position they will have in $C^{t_{1}}$.

It is easy to see that in the model so described edge effects will arise, since spontaneous shrinking will concern boundary elements because of the non-symmetric placement of their neighbors. In order to overcome this problem several standard possibilities can be considered. Choosing probably the most simple one, we introduce a 'fictitious' boundary constituted by an external frame of elements which simply follow, at a fixed distance and always in the same direction, their closest 'true' element. The fictitious elements move only in a specific virtual time step which follows the other ones. In this way, every true element will have a complete set of 8 neighbors (see Fig. 3 for a graphical representation. An explicit description of the motion of the fictitious elements is in [6]). The distance at which the fictitious elements are positioned represents a key characteristic length, determining the lattice step of the system in the equilibrium configuration. In the following numerical simulations, unless differently specified, this length is equal to 1 length unit for all the fictitious elements.

The previous algorithm can be easily generalized to the case of second and, in general, $n$-th neighbors interaction, by simply computing the centroid of sets of points having maximum Lagrangian Chebyshev distance of 2 (in general, $n$ ). In this case, additional sets of fictitious elements have also to be introduced in order to have, for every element of the system, a full set of neighbors. In [6] the system is also gen- 


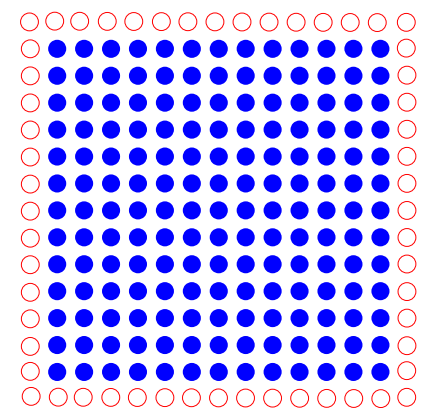

Fig. 3 Graphical representation of fictitious elements. Every fictitious element (empty red dots) moves in rigid translation with the closest true one (blue dots); every vertex true element carries in rigid translation the vertex fictitious one and also its two fictitious neighbors.

eralized to the case of multiple leaders, which will be used throughout the present paper.

The geometric centroid of a given set of points $P_{1}, \ldots, P_{n}$ minimizes the sum of the squared distances, i.e. the function:

$$
f(P)=\sum_{i=1}^{n}\left\|P_{i}-P\right\|^{2}
$$

It is therefore possible to see that for a first-(second-)neighbor interaction, a natural deformation energy density can be written as a function of first-(second-)order finite differences of the placement $\chi$ for a given point, and therefore, as the step length goes to zero, a second gradient homogenized energy $\mathscr{E}[\nabla \chi(P), \nabla \nabla \chi(P)]$ can be conjectured for second neighbors interaction systems (for more details see again [6]).

The algorithm described before has much in common with theoretical models conceived for describing collective behaviors (e.g. the evolution of swarms, flocks etc.). Such models have already been used for mechanical investigations, in cases in which the potential energy of a mechanical system is minimal in correspondence with stable equilibrium positions of a swarm system $[38,39]$. However, in the present work we prefer not to use the name 'swarm' system, since one usually associates that name, and in particular the expression Particle Swarm Optimization (PSO), to cases in which each particle of the swarm represents a potential solution of a given (a priori formulated) optimization problem [40, 41, 42]. Our aim, in fact, is not the study of numerical tools for the solution of well defined optimization problems, but rather the development of a new model directly accounting for the description of the phenomenology; other features distinguishing the proposed discrete model from PSO ones is the presence of leaders (whereas PSO models are usually anarchic) and the absence of any randomness. 
It should also be pointed out that some basic features of our model (in particular the Lagrangian character of the neighborhood) are quite different from standard swarm robotic modeling. In modeling swarms, flocks and schools dynamics, a topological concept of neighborhood (rather than a metric one) is emerging as one of the most promising in order to account for the observed phenomenology (see [43, 44]). The model proposed in the present paper mixes these two ideas, since it is based on a concept of neighborhood which depends on the topological distance between the elements, while the way in which the interaction works depends on the metric (Euclidean) distance in the actual configuration. Finally, another feature of the proposed model which is often met in swarm modeling is that the elements, due to the presence of the fictitious boundary, do not behave all in the same way; this has been proposed as one of the possible discriminating factors between crowds and swarms/flocks/schools models (see e.g. [45, 46]), but also in this case it can be pointed out that the property of being (or not) a boundary element, in the model considered herein, is Lagrangian, i.e. it is preserved during the time evolution of the system.

\subsection{Short summary of previous numerical results}

We summarize in this section the numerical results obtained in [6]. With the aim of comparing the discrete models characterized by (respectively) first and second neighbor interaction (FNI and SNI) with first and second gradient continuum theories, some simple cases were investigated.

Two squared systems FNI and SNI were considered, in which the leaders $\mathfrak{L}$, situated in a vertex of the square, were pulled outside (or pushed inside) in the diagonal direction at 45 degrees with respect to the sides of the system in $C^{*}$ with a uniform motion. The numerical results obtained were compared with Finite Element simulations of a 2D continuous squared body, at the vertex of which a prescribed displacement was imposed. Two cases were considered: a standard energy (for the first gradient case) and the Mindlin general form [15] for the second gradient case. In Fig. 4 we can appreciate one of the similarities between the continuum models and the discrete system here described (for other features see [6]). As we can see, in the FNI/first gradient continuum cases a loss of boundary convexity around the vertex is observed, whereas this behavior is not present in SNI/second gradient continuum cases; this is highlighted in Fig. 5. Releasing the vertex at a certain time step, i.e. letting the system evolve while the leader is stopped, one observes the return to the original configuration, in agreement with the behavior expected in elastically deformed bodies. As can be seen in Fig. 6, the return to the initial configuration is not instantaneous, which implies the presence of inertia effects even if no explicit variable accounting for the elements mass was introduced. It is possible to prove that actually we have an asymptotic convergence, which means that some viscous effect has to be considered if one want to identify the Lagrangian system exactly corresponding to the evolution of the proposed system ([6]). 


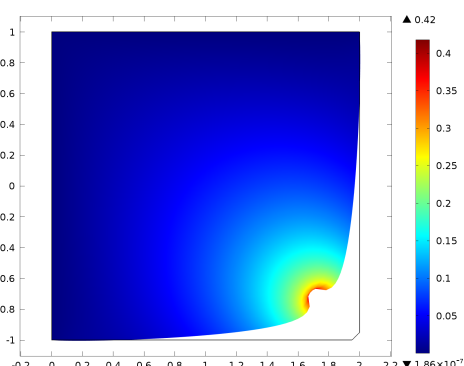

(a)

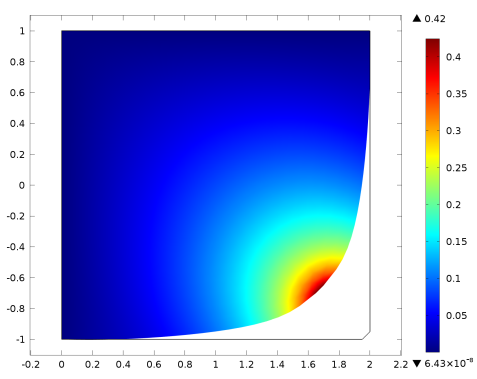

(b)

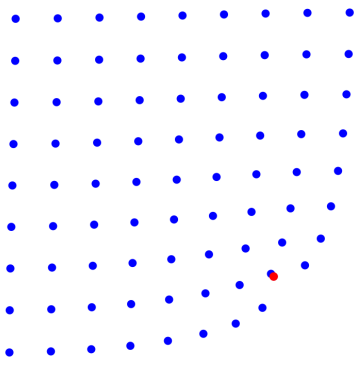

(c)

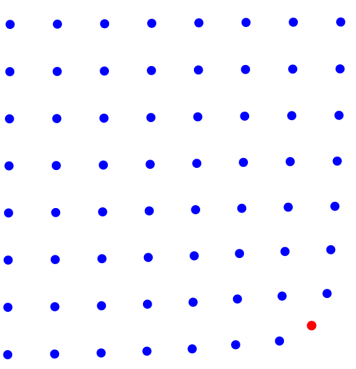

(d)

Fig. 4 A comparison between FE simulation of first (a) or second (b) gradient continua and first (c) or second (d) neighbors interaction with a similar external action.

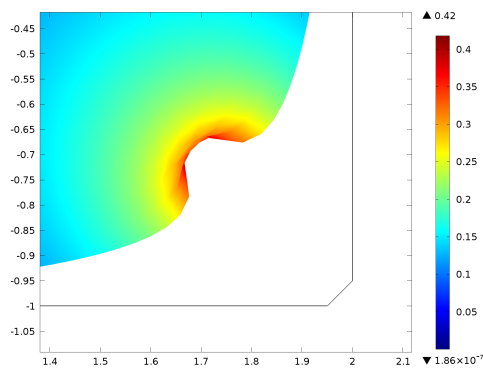

(a)

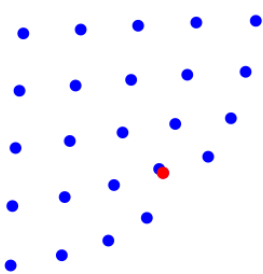

(b)

Fig. 5 Zoom on the loss of convexity in first gradient (a) and first neighbors interaction (b) cases. 


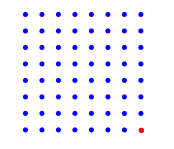

(a) $t=1$

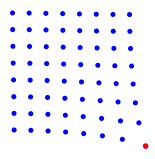

(d) $t=300$

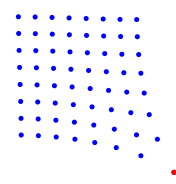

(b) $t=100$

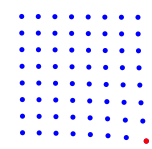

(e) $t=400$



(c) $t=200$

$::::::$

$::::::$

$:::::: 2$

$: \geq: \geq:$

(f) $t=500$
$:::::::$
$\because:: \geq::$
$\because:: \geq: 2$
$: \geq:: 2:$
$\because:::::$
$\because: 2: 2$
(g) $t=600$
(h) $t=700$

Fig. 6 Imposing a stop to the leader (at $t=150$ ) the system tends to return to the original configuration.

These results (among others) suggest that the evolution of the presented discrete system resembles that of elastic deformable bodies, and that varying the order of the interaction, specific characteristics of higher gradient theories are also recovered. In the following we will see a more direct comparison with 2D continuum simulations performed with COMSOL.

Finally, we want to devote a few words to the nonlinear character of the evolution of the system. In the following sections, a fracture algorithm (obviously entailing nonlinearities) will be introduced, but even the basic form of the model as described up to this point exhibits a nonlinear behavior. In Fig. 7, we compare two identical systems in which different actions are imposed to one single leader. In the left panel, a speed of 1 length units per unit time is imposed for a total of 10 time steps; in the right panel, we imposed a speed of 0.001 length units per unit time for a total of 10000 time steps. As one can see, the resulting configurations are clearly different, thus implying the nonlinearity with respect to the imposed external action. 


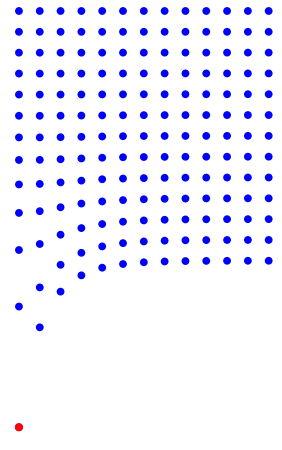

(a)

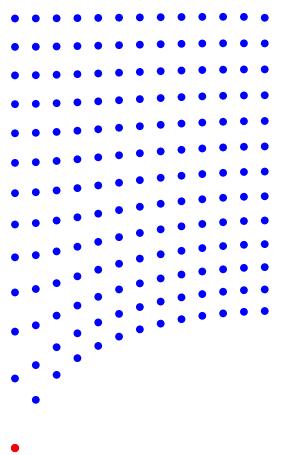

(b)

Fig. 7 Nonlinearity of the system: in (a), a speed $s=1$ length units per time step is imposed to the leader for an interval $I$ of 10 time steps; in (b), the $s=0.001$ and $I=10000$. The two resulting configurations are different.

\subsection{Further comparison with Finite Element simulations}

In this section we want to directly compare the results of our discrete model with the ones obtained by standard Finite Element simulations.

In Fig. 8 we show the superposition of:

- a simulation with an imposed external action consisting in pulling in opposite directions two opposed vertexes of our system;

- a standard FE simulation (performed with COMSOL) in which a similar action (imposed displacement) is applied on a squared 2D continuum.

For the continuous simulation, we considered both a classical Cauchy continuum (left) and a second gradient one (right). In color map the modulus of the displacement is shown. The corresponding discrete simulation involve first- (left) and second-neighbors (right) interaction. As it can be seen, in both cases the shape of the sample is accurately approximated by the discrete system, in the specific case employing only a very limited number of elements $(12 \times 12=144$ elements in total $)$. This means that our system produces reliable results with a very limited computational cost, at least with this kind of external action.

\section{Spontaneous evolution and Discrete Energy Equivalent}

The previously described algorithm can be adapted to the case of a spontaneously evolving system, i.e. systems in which we do not impose a particular motion to any 


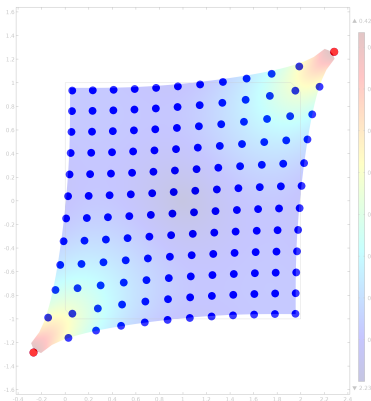

(a)

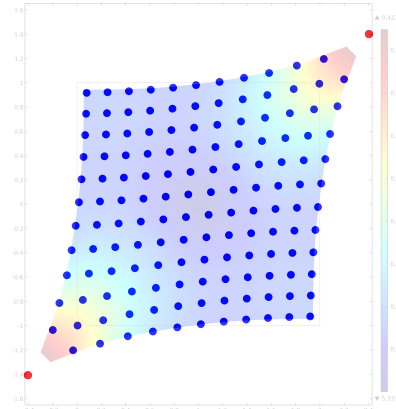

(b)

Fig. 8 Superposition of the discrete system with a standard FE continuum simulation involving first gradient/first-neighbors (left) and second gradient/second neighbors models (right).

of the elements. To do so, we start from an arbitrary initial configuration $C^{0}$, in which in general the elements do not lie in the centroid of their neighbors. We apply the algorithm starting from a selected element $\mathscr{P}$ (which we will call the 'pseudoleader') and then proceeding through concentric square frames. The only difference with the case of a 'true' leader is that the pseudo-leader $\mathscr{P}$ does not obey to an imposed motion, but simply goes, in the first virtual step, in the centroid of its first neighbors as all other elements do.

The observations made at the end of section 1.2 suggest the introduction of a quantity $\mathscr{E}^{D}(t)$, which we will call Discrete Energy Equivalent (DEE), defined on a geometrical basis. The DEE will represent a measure of the deformation energy stored in the actual configuration of the system in case of first neighbors interaction. Due to the minimum property of the centroid above recalled (see Eq.(1)), and considering that an (Euclidean) distance equal to 1 or to $\sqrt{2}$ corresponds to the pairs of first neighbors elements in the equilibrium configuration $C^{*}$, it is natural to define $\mathscr{E} D$ as follows:

$$
\mathscr{E}^{D}=\sum_{S_{1}}\left(d^{t}-1\right)^{2}+\sum_{S_{2}} \frac{\left(d^{t}-\sqrt{2}\right)^{2}}{2}
$$

Here the sums are extended over all the pairs of first neighbors 'true' elements. The sets $S_{1}$ and $S_{2}$ contain the pairs that in $C^{*}$ lie respectively orthogonally or diagonally with respect to the sides. A unit length is subtracted from $d^{t}$ ( $\sqrt{2}$ in case of diagonal pairs) because the step length is unitary; more precisely, because the fictitious elements are at a fixed unitary distance from the true boundary. Since the sums have the dimension of an area, a coefficient $\alpha$ having the dimension of a force unit over a length unit has to be introduced to define the energy of the system: $\mathscr{E}=\alpha \mathscr{E}^{D}$. 
The first set of numerical simulations will concern the study of the DEE decay in systems evolving without leaders, and since in our simulations $\mathscr{E} D\left(C^{0}\right) \neq 0, C^{0}$ can be seen as a pre-stressed configuration. A basic case, in which the center element is selected as the pseudo-leader, is represented in Fig. 9. A sharper case in which the initial configuration is more stressed is shown in Fig. 10. In both cases, the energy decay is well interpolated by an exponential behavior; in Fig. 11 the interpolation of the form $e^{a x^{b}}$, with $a \approx-0.59$ and $b \approx 0.52$ is shown. This behavior is well known for several elastic systems in which viscous dissipation occurs in both linear and nonlinear cases (see e.g. [47, 48, 49, 50]).

One may wonder whether the system always asymptotically converges to the same limit configuration (i.e., the one with $\mathscr{E}^{D}=0$ ) independently on the choice of the pseudo-leader. This seems a rather natural request if the system is intended to model the behavior of a deformable body. In the Appendix (see 4) we prove that in the 1D case the answer is yes. In 2D, the conjecture that the asymptotic configuration is independent on the pseudo-leader choice remains more than reasonable, but the proof is more difficult, since in this case it is not true anymore that the total discrete energy decreases in every virtual step. Numerical evidence of this somewhat surprising statement is shown in Fig. 12, where one can observe that, after a first approximately exponential phase, the energy increases slightly before its eventual decay.

We want now to perform some quantitative analysis in order to evaluate some magnitudes concerning the evolution of our system on the basis of the DEE above defined. Let us first consider a very simple case in which uniaxial motion is imposed to a set of leaders constituted by the elements belonging to two opposite sides of the system. In particular, the fictitious elements relative to the left side are treated as leaders, as they are motionless by definition (from a mechanical point of view, the side has an imposed displacement equal to zero). Moreover, we impose a motion to the elements belonging to the right side; the motion is uniform and directed along the $x$ axis up to a certain time $t_{s}$, after which they stop. The system is then left evolving until equilibrium is achieved. The result, with relative energy versus time plotting, is shown in Fig. 13 (here and in all the following simulations, the leaders are represented by red dots). Since our external action is an imposed motion, it is not immediate to derive from it a discrete version of the applied uniaxial force $\sigma^{D}$. We can however reasonably define it in various equivalent ways; for instance, we can use the identity

$$
\frac{\left(\sigma^{D}\right)^{2} A}{2 E}=\mathscr{E}=\alpha \mathscr{E}^{D}
$$

where $A$ is the area of the system at the equilibrium, $E$ is Young's modulus and $\alpha$ the previously introduced constant. We will not lose generality by selecting the unit for $\sigma^{D}$ so as to have $2 E=1$, and therefore we get $\left(\sigma^{D}\right)^{2}=\frac{\alpha \mathscr{E}^{D}}{A}$. The numerical data for $A$ and $\mathscr{E}^{D}$ give:

$$
\left(\sigma^{D}\right)^{2}=\frac{\alpha \mathscr{E}^{D}}{A} \approx \frac{12.5 \alpha}{382} \approx 0.03 \alpha
$$

Using the reasonable assumption that a certain imposed motion will approximately correspond to the same $\sigma^{D}$ if only small and local changes are considered in $C^{*}$, we 


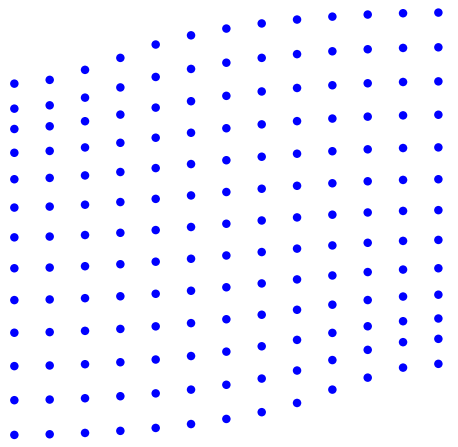

(a) $t=1$

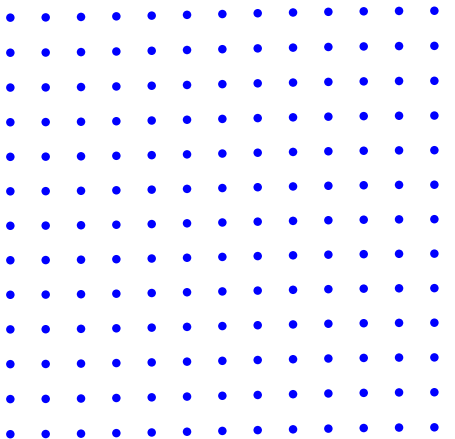

(c) $t=60$

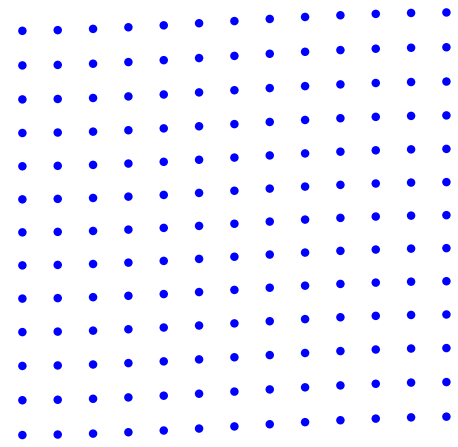

(b) $t=30$

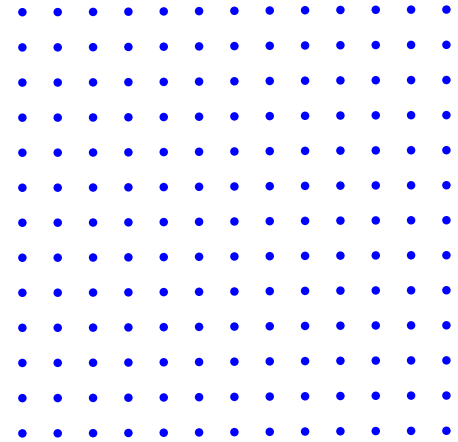

(d) $t=100$

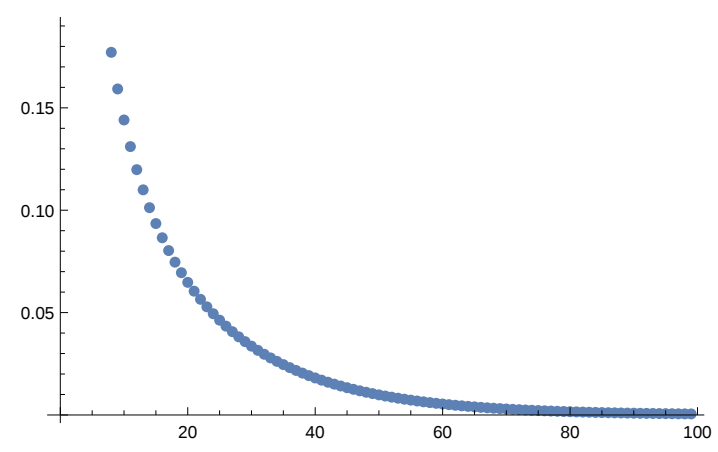

(e)

Fig. 9 (a-d): spontaneuos evolution of the system from a prestressed configuration; the pseudoleader is the central element. (e): time history of the Discrete Energy Equivalent 


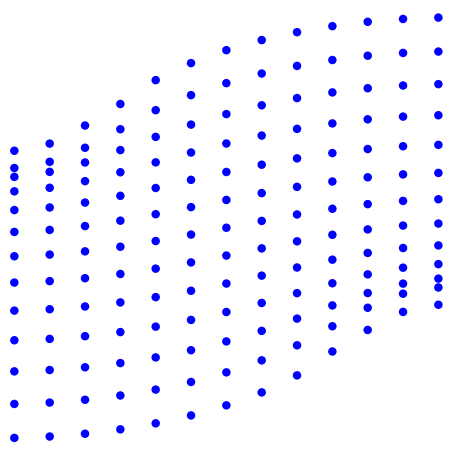

(a) $t=1$

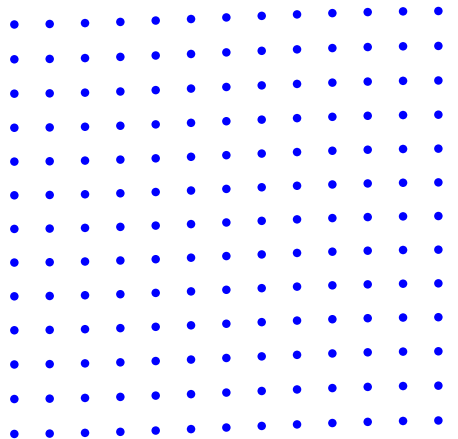

(c) $t=60$

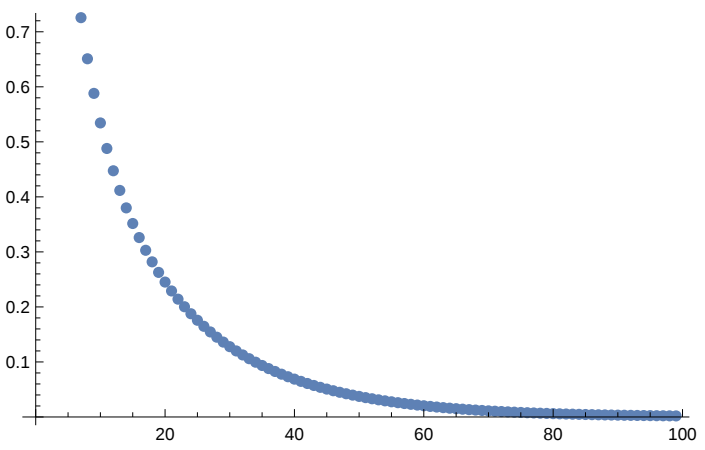

(e)

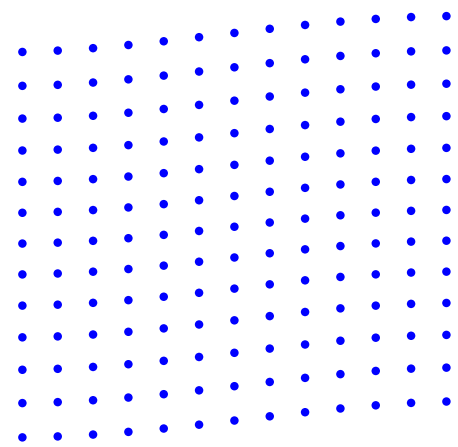

(b) $t=30$

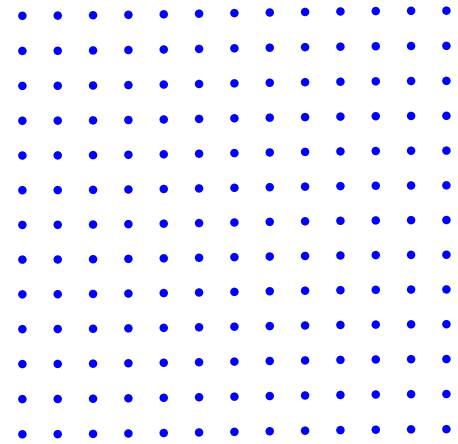

(d) $t=100$

Fig. 10 (a-d): the same simulation of Fig. 9 with a more stressed initial configuration. (e): time history of the Discrete Energy Equivalent 


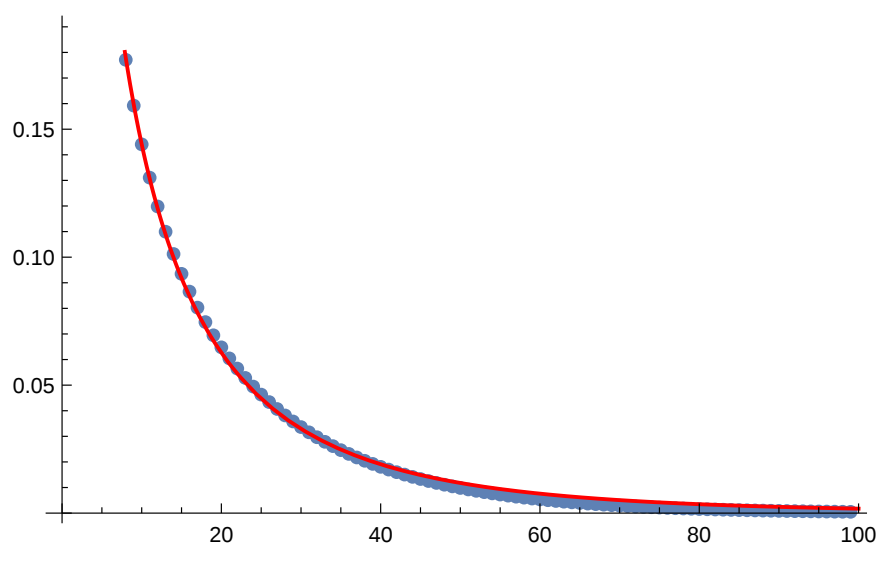

Fig. 11 Exponential energy decay: in red it is represented the curve $e^{-0,5912 x^{0,5155}}$.

will employ the value now obtained in the simulations on the crack formation and growth considered below.

\section{Fracture and crack formation and evolution}

\subsection{Introduction of the fracture}

In the proposed model, the fracture is intended as a loss of interaction between neighboring elements. Specifically, when the Euclidean distance (evaluated in actual configurations) between two interacting elements overcomes a certain threshold $s_{f}$, the two elements do not interact anymore. Obviously, when this happens, the computation of the centroid relative to the considered elements is ill defined, in the same sense as intended for what concerns boundary elements, as seen in section 1.2. We solve the problem in a similar way as done before. Indeed, we introduce for every bond break a new pair of fictitious elements evolving in the same way as the fictitious boundary elements introduced above, i.e. each of them following one of the two elements whose bond has been broken. In this way, the two elements among which the fracture occurred become boundary elements in the same sense as the preexisting ones.

The fracture algorithm works as follows: we introduce a prescribed order in the $n$-th neighbors of a given non-fictitious element starting, for example, from the neighbors in the left upper corner of the frame square centered in the considered element and proceeding clockwise. The labeling of neighbor elements allows the introduction of an adjacency matrix $J$, that in this case is a tensor of boolean variables $J_{i j k}$, the first two indexes determining the particle $(i, j)$ of the system, and the third one $k$, ranging from 1 to 8 in FNI, identifying one of its neighbors. When the distance 


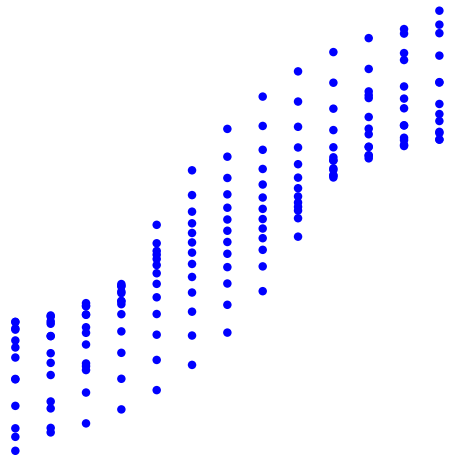

(a) $t=1$

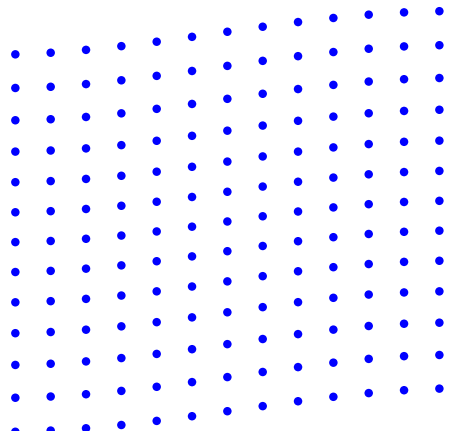

(c) $t=60$

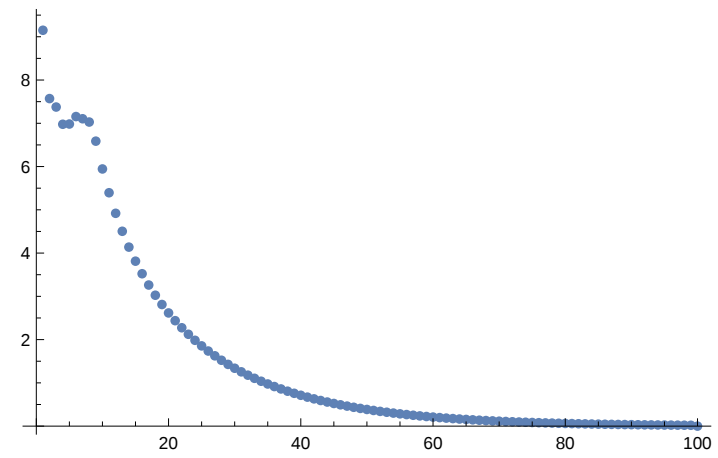

(e)

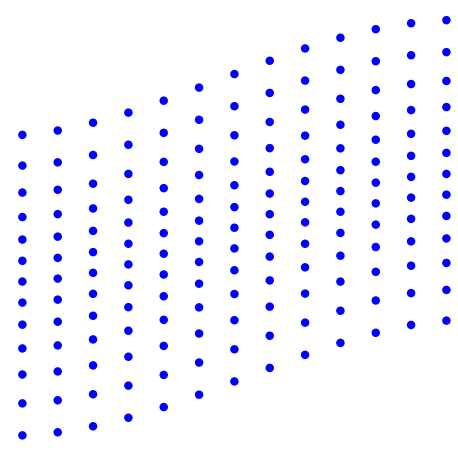

(b) $t=30$

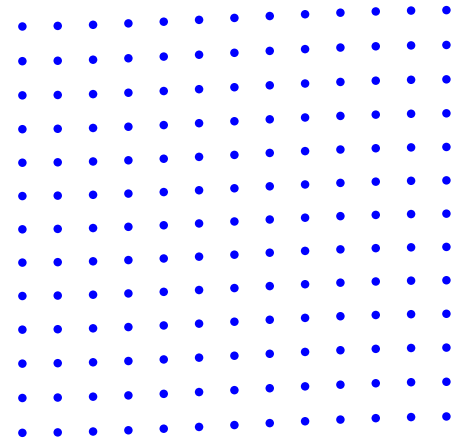

(d) $t=100$

Fig. 12 Spontaneous evolution starting from a severely prestressed configuration: the energy is not a monotonically decreasing function. 




(a) $t=1$

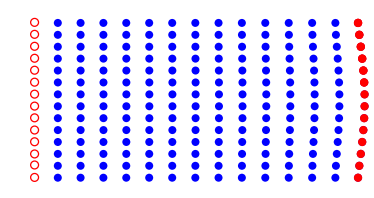

(c) $t=660$

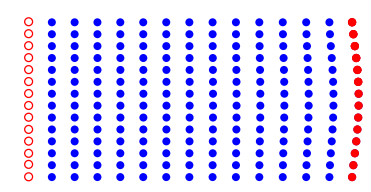

(b) $t=330$

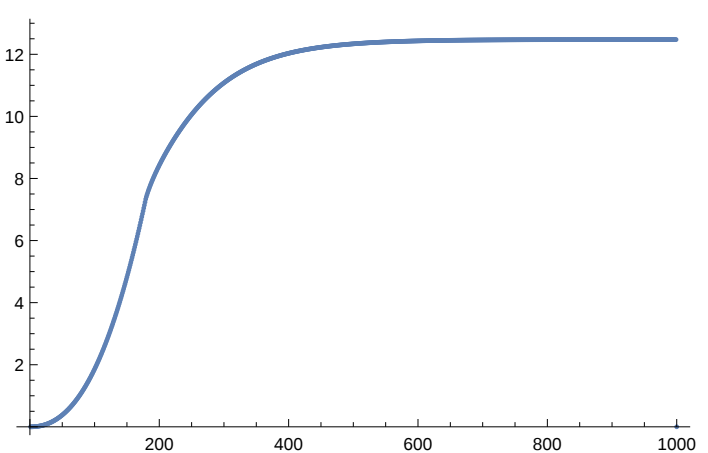

(e)

Fig. 13 Uniaxial imposed motion to the leaders. The leaders (solid red dots) have a uniform motion parallel to the $x$ axis and then are stopped at $t=180$. An equilibrium configuration is reached around $t=450$. We use empty red dots to indicate that the fictitious elements relative to the left side have an imposed null velocity, i.e. the left side has an imposed displacement equal to zero.

between two elements is larger than $s_{f}$, the value of the corresponding element of $J$ is set to $F A L S E=0$ (otherwise it is TRUE=1). When the centroid is computed, each neighbor coordinate is multiplied by the relative element of $J$. Moreover, another term is considered in the centroid computation, consisting in the coordinate of fictitious elements, each multiplied by $\bar{J}$ (where the bar indicates logical complement). It is clear that in this way, when the centroid is evaluated, a true or a fictitious element enters the calculation according to the fact that the interaction is present or broken. The irreversibility of the fracture, which is a desirable feature when modeling solid bodies, is verified since once an element of $J$ is set to 0 , it can not become 1 anymore.

Another degree of freedom of the model, concerning the fictitious elements appearing after the fracture, naturally emerges form the described algorithm. Indeed, one 
can choose to place the new fictitious elements at a distance $\rho_{f}$ that does not correspond to the lattice step in $C^{*}$ (more precisely, we should say that it would not correspond to the fixed distance at which the fictitious boundary is from the true elements. It is indeed this last distance that determines the lattice step at which the system is in equilibrium). In this way, as we will see in the following, relevant features of fracture phenomena can be modeled.

\subsection{Basic fracture and crack evolution}

A well known variational approach to the fracture was developed by Griffith in the '20. From Griffith's model (in 2D case), we have at the equilibrium:

$$
\mathscr{E}_{T O T}=2 \gamma a+\frac{\sigma^{2}}{2 E} A-\frac{\sigma^{2}}{2 E} \beta a^{2}
$$

where $\gamma$ represents the energy per unit line required to break atomic bonds, $E$ the Young Modulus, $A$ the area of the sample, $a$ is the crack length, $\sigma$ the stress and $\beta$ an non-dimensional parameter accounting for the measure of the part of the surface relaxing as a consequence of the crack opening. The most relevant qualitative aspect of Griffith's theory is that the dependence of the energy on the crack length forecasts that below a critical value of $a$ the system is stable, and a crack growth is possible only providing additional energy to the system. If this critical value is reached, the system becomes unstable and the crack evolves spontaneously. In the next groups of simulations we selected two leaders belonging to the bottom side (red elements) and imposed to them a uniform velocity, with components $(0.03,0.03)$ and $(-003,0.03)$ respectively. The leaders were stopped at $t=80$. In Fig. 14 we set the fracture threshold $s_{f}=1.17$ length units; we can see that the crack keeps growing even after the leaders have stopped, that means that the crack length is reaching the equilibrium value for the given imposed action. The crack length equilibrium value is reached around $t=160$, after which the cracks remain stable while the system keeps relaxing and releasing deformation energy.

In Fig. 15 the same simulation was performed with the fracture threshold set at $s_{f}=1.12$. It can be seen that the crack growth does not stop and indeed goes up to the complete split of the system, which is reached around $t=250$. This means that stopping the motion at $t=80$ is sufficient for furnishing to the system the energy needed to reach the length threshold after which the crack grows spontaneously.

\subsection{Uniaxial external action}

The previous results indicate that qualitative features of Griffith's theory are recovered through our discrete model. However, in order to be able to compare quantitatively our numerical results with Griffith's theory, we need to apply a simpler 


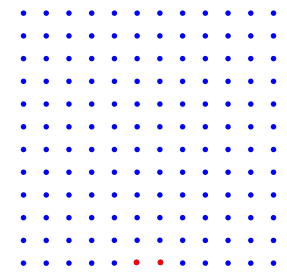

(a) $t=1$

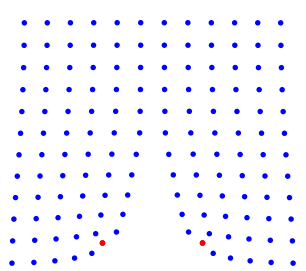

(c) $t=160$

$\cdots \cdots \cdots$

$\therefore \therefore \therefore \therefore \therefore \therefore$

$\cdots \cdots$

:

........

$\cdots \cdots$

$\cdots$

$\ldots \ldots \ldots$

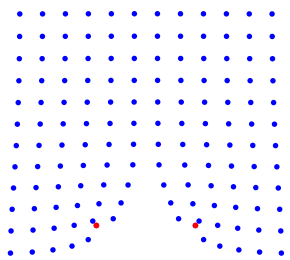

(b) $t=80$

$\because \cdots \cdots$

$\therefore \cdots \cdots$

$\therefore \therefore \cdots$

$\ldots: \cdots \cdots$

$\therefore \ldots$

$\because \because \cdots$

$\because \because \because \cdots \cdots$

$\ldots \ldots$

$\therefore \therefore \because \quad \because \ldots \ldots$
$\ldots \ldots$
$\cdots \cdots$

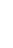

(e) $t=1000$

(f) $t=2000$

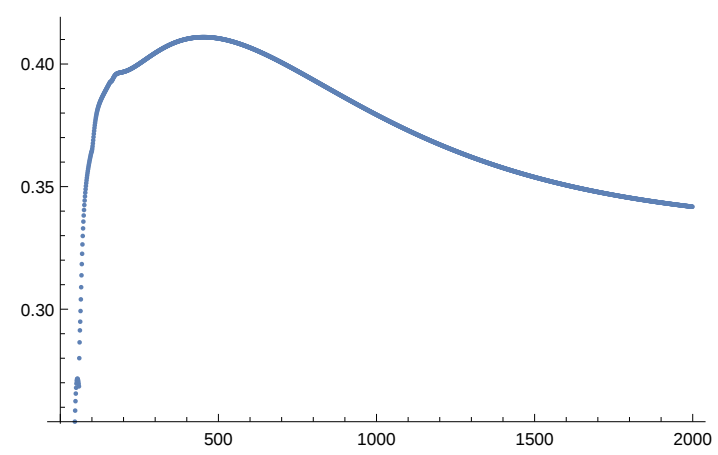

(g)

Fig. 14 Crack growth as a consequence of two leaders pushing from inside at 45 degrees with uniform velocity; with $s_{f}=1.17$ the crack length stabilizes around $t=160$. 


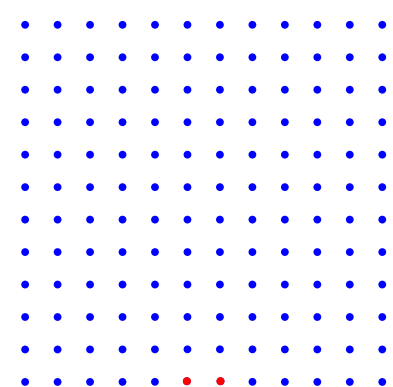

(a) $t=1$

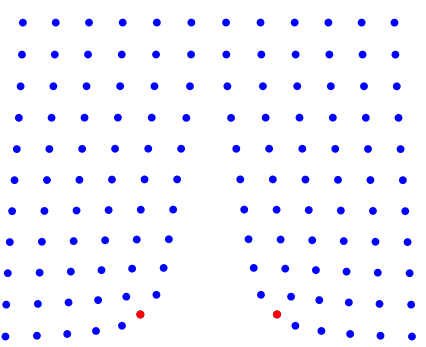

(c) $t=200$

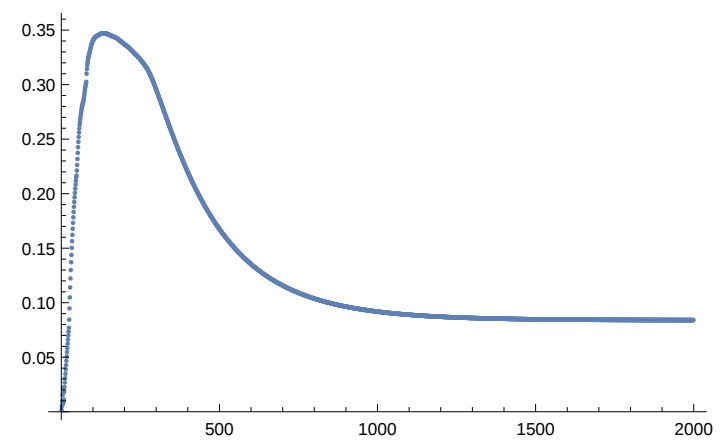

(e)

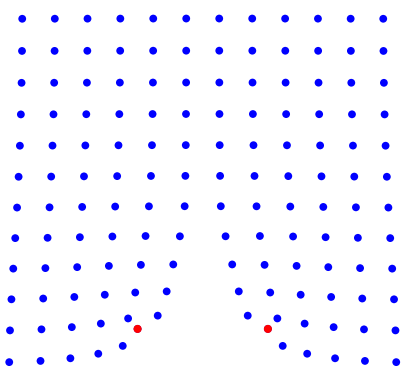

(b) $t=100$

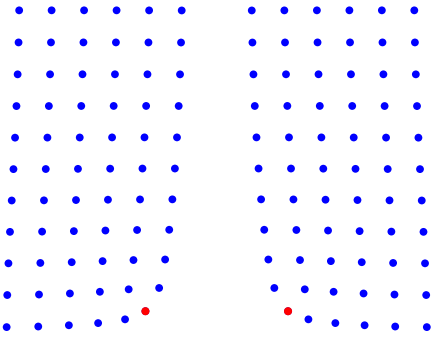

(d) $t=300$

Fig. 15 The same simulation of Fig. 15 is performed with $s_{f}=1.12$; in this case, the critical length is overcome and the crack growth keeps up to the complete split of the system. 
external action, i.e. a uniaxial one, as done in the simulation shown in Fig. 13. Let us first formulate Eq.(4) by means of the discrete variables considered here. In our model, the energy can be written as:

$$
\mathscr{E}_{T O T}=3 \alpha\left(s_{\text {eff }}\right)^{2} l+\left(\sigma^{D}\right)^{2} A-\left(\sigma^{D}\right)^{2} \beta l^{2}
$$

where $s_{\text {eff }}=s_{f}-1$ is the DEE lost when a single bond is broken, $A$ represents the area of the system (in squared unit lengths) and $l$ is the number of broken pairs of elements in the crack.

In the simulation shown in Fig. 16 we imposed to the leaders the same external action used in the simulation of Fig. 13, i.e. we imposed an uniaxial displacement of the leaders in the $x$ direction up to 180 time steps followed by a stop, and a zero displacement to the fictitious elements relative to the left vertical side. In order to see a crack formation and evolution we created a 'defect' by removing 6 bonds close to the middle points of the bottom side. We can observe that a crack indeed opens in correspondence with the defect, and that it reaches a stable length already at $t=1000$.

The DEE $\mathscr{E}^{D}$ that we obtained as an output $(\approx 5)$ times the dimensional constant $\alpha$ is the discrete estimate of the deformation energy of the system, and therefore represents the quantity given by the last two terms in the right hand side of Eq.(5). This allows us to provide an estimate for $\beta$. Indeed, substituting the value for $\sigma^{D}$ taken from the simulation shown in Fig. 13, we have:

$$
\beta=\frac{\left(\sigma^{D}\right)^{2} \mu n^{2}-\alpha \mathscr{E}^{D}}{\left(\sigma^{D}\right)^{2} l^{2}} \approx 25.7
$$

According to the standard interpretation in Griffith's fracture theory, this means that an area measuring approximately $\beta \times l^{2}=25.7 \times 9$ is relaxed as a consequence of the crack formation; this area corresponds to a fraction of approximately 0.58 of the total area.

Let us now consider the first term in the right hand side of Eq.(5). The numerical output tells us that a total of 9 bonds, in Fig. 16, are stretched beyond the fracture threshold (see Fig. 17 for a graphical representation). As we recall, we introduced a 'defect' consisting in removing six interactions close to the edge; this means that a total of three elementary bonds were broken by applying the external action. Since the threshold was set at $s_{f}=2.62$, the corresponding DEE, i.e. the energy $\mathscr{E}_{b}$ required to break the bonds can be estimated by:

$$
\mathscr{E}_{b}=\alpha\left(s_{f}-1\right)^{2} \times 6=7.68 \alpha
$$

The sum $\mathscr{E}_{b}+\alpha \mathscr{E}^{D} \approx 12.68 \alpha$ is an estimate of the total energy in the system. As one can see, it is very close to the total deformation energy $(\approx 12.5 \alpha$, see Eq.(3)) with the same external action in case no fracture threshold is considered. This is consistent since, in the proposed model, no additional dissipated energy is associated to the break of the interactions, and therefore the total energy should coincide with the deformation energy. The small difference has to be related with the fact that we 


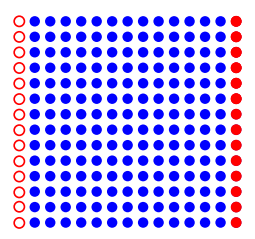

(a) $t=1$

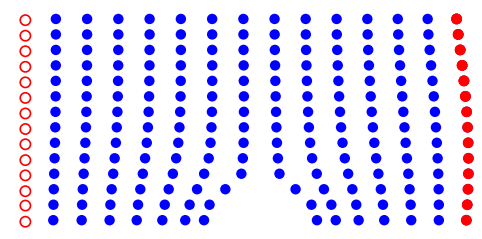

(b) $t=1000$

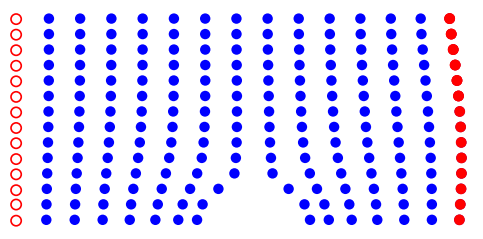

(c) $t=2000$

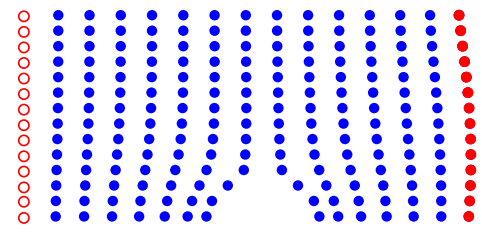

(d) $t=3000$

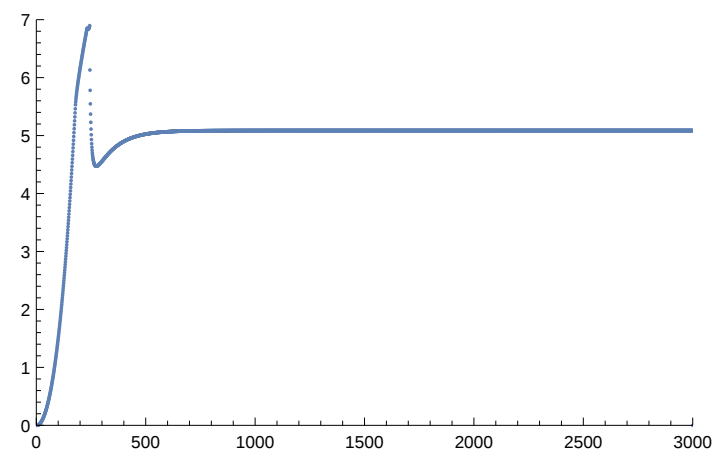

(e)

Fig. 16 Uniaxial external action imposed to a set of boundary leaders with a defect consisting in the removal of six bounds around the middle elements of the bottom side $\left(s_{f}=2.62\right)$. The leadres stop at $t=180$; a crack opens in correspondence with the defect and reaches a stable length (as before, we use empty red dots to indicate that the fictitious elements relative to the left side have an imposed null velocity, i.e. the left side has an imposed displacement equal to zero). 
introduced a local defect in the simulation shown in Fig. 16; since we imposed an external action consisting in imposed displacements, this means that, assuming the same value for $\sigma^{D}$ as the one measured in the simulation of Fig. 13, we are slightly overestimating it. Actually, the ratio $\frac{12.68-12.5}{12.68} \approx 0.014$ is very close to the ratio between the number of removed bonds over the total bonds present in the system $\left(\frac{6}{520} \approx 0.012\right)$.

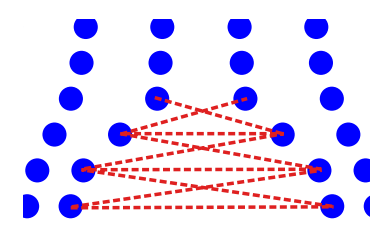

Fig. 17 Graphical representation of the bonds broken in the simulation shown in Fig. 16.

\subsection{Almost-steady state}

In the next simulation we want to underline a peculiar behavior shown by the model which can be called as an 'almost steady' state followed by a catastrophic evolution of the crack. We considered the same system of the previous one, making only a small change in the fracture threshold $s_{f}$. Tuning very finely the threshold (i.e. setting $s_{f}=2.5741$ ), one can obtain that a significantly long almost steady phase is followed by a nearly spontaneous crack opening around 1000 time steps (Fig. 18). We recall that the leaders were stopped at $t=180$, which means that the abrupt opening of the crack around $t=900$ is a consequence of an internal evolution of the system, though it is hardly visible since only very tiny changes in the positions of the elements (virtually invisible at the 'macro' level) occur for a long time. In the opinion of the authors, this behavior, which is experimentally well known [51], is quite interesting, especially considering the simplicity of the discrete model employed. The sensitivity of the model with respect to very fine changes in the parameters suggests that non trivial instability issues may arise from the study of its homogenized form. This can be a very interesting aspect to be investigated, useful theoretical tools being available in recent literature (the interested reader can see, for instance, $[52,53,54,55,56])$

\section{Conclusion and further objectives}

In this work a new simple discrete system (introduced in [6]) with a centroid-based propagating interaction has been used in order to account for fracture phenomena in deformable bodies. Some results on pure deformation including a direct comparison 


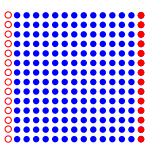

(a) $t=1$

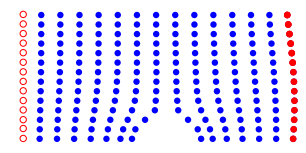

(c) $t=530$

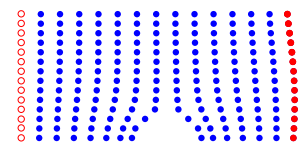

(e) $t=860$

(g) $t=1030$

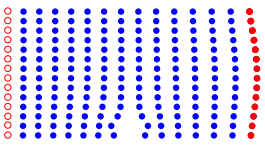

(b) $t=215$

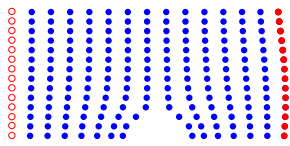

(d) $t=745$

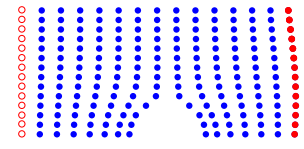

(f) $t=980$

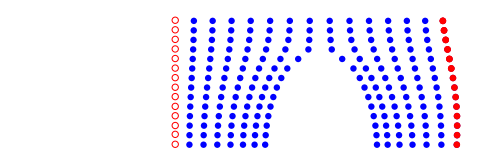

$$
\text { (g) } t=1030
$$
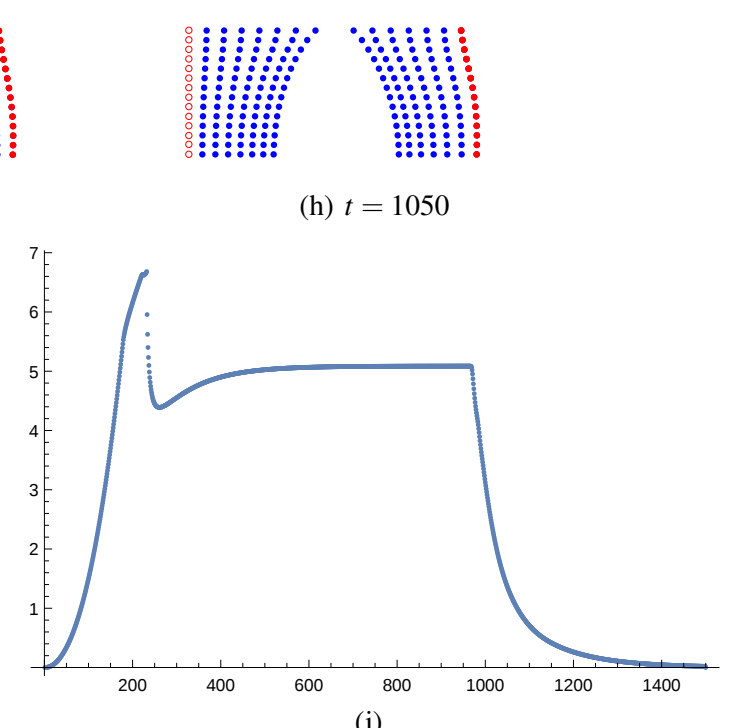

(h) $t=1050$

(i) $t=1075$

(j)

Fig. 18 'Almost steady' state followed by a quick and spontaneous evolution of the crack; $s_{f}=$ 2.5741 , leaders are stopped at $t=180$. Between $t \approx 500$ and $t \approx 1000$ the energy plot is almost flat, then an abrupt crack opening occurs (as before, we use empty red dots to indicate that the fictitious elements relative to the left side have an imposed null velocity, i.e. the left side has an imposed displacement equal to zero). 
with standard Finite Element simulations are presented. A discrete equivalent of the deformation energy is also defined and used to prove the independence of the asymptotic configuration on the choice of the element from which the algorithm starts in case of spontaneous evolution. A fracture algorithm is then introduced, with the definition of a suitable adjacency matrix specifying the pairs of elements among which no interaction exists anymore because the two elements overcame a prescribed threshold in the actual distance. The discrete equivalent of an external stress is also defined in the uniaxial case, and used to compare the numerical results with Griffith's theory of fracture. Different examples of crack formation and growth are considered.

The results indicate that the proposed model is promising for developing new and computationally advantageous tools for the study of fracture, and that the model is rich enough to produce interesting behaviors such as a spontaneous crack evolution after a long almost steady state.

Future investigations will concern how, by suitably weighting the contribution of the neighbors in the computation of the centroid, one can obtain different material models, and in particular different kinds of anisotropies. Moreover, one may try to generalize the model by suitably coupling the coordinates in order to account for Poisson's ratios different from 0 (while using directly the centroid-based algorithm we described this is the only possible value). Also interesting will be the study of more cases in which higher order interactions, reproducing behaviors typical of higher order continua, are considered. Finally theoretical results, among which the investigation of the homogenized limit of the considered system and the relative variational formulation, are needed in order to make further and sound progresses in the subject.

\section{Proof of the independence of the asymptotic behavior on the choice of the pseudo-leader for $1 D$ systems}

In Section 2 we considered the problem of the uniqueness of the asymptotic configuration of the system evolving spontaneously (i.e., without leaders), independently on the selected pseudo-leader. As this represents an important objectivity requirement for the model, in the present appendix we deserve a more formal treatment to the $1 \mathrm{D}$ case.

Let us consider a 1-dimensional system $\mathfrak{S}$ constituted by $N$ elements mathematically represented by points in the real line, and an infinite set of discrete time steps $\mathfrak{T}=\left\{t_{0}, t_{1}, \ldots, t_{m}, \ldots\right\}$. The system will evolve exactly as explained in the $2 \mathrm{D}$ case, with the set of first neighbors being constituted, for every element, by the two closest ones. We call configuration of $\mathfrak{S}$ a strictly increasing function $C$ mapping $i$ into $x_{i} \in \mathbb{R}$, with the integer $i: 0<i \leq N$ indicating the Lagrangian label of the elements of the system. We indicate by $C^{t_{j}}$ the configuration at time $t_{j} \in \mathfrak{T}$, and by $\mathfrak{S}_{\bar{i}}$ the system evolving with the chosen pseudo-leader $(\bar{i})$. We say that $\mathfrak{S}_{\bar{i}}$ asymptotically converges to the configuration $C^{t_{\infty}}$, and write $\mathfrak{S}_{\bar{i}} \rightarrow C^{t_{\infty}}$, if for every $\varepsilon>0$ there ex- 
ists a positive integer $M$ such that $\left|x_{i}^{t_{m}}-x_{i}^{t_{\infty}}\right|<\varepsilon$ for every integer $i: 0<i \leq N$ and every $t_{m} \in \mathfrak{T}: m>M$ (we can notice that the given definition, which is natural when considering finite systems, would correspond in the homogenized limit to a uniform functional convergence). Let $\mathfrak{C}^{*}$ be the set of configurations in which $\left|x_{i+1}-x_{i}\right|=1$ for every integer $i$ such that $0<i<N$; obviously one has $\mathscr{E}^{D}\left(C^{*}\right)=0$ for every $C^{*} \in \mathfrak{C}^{*}$ (see Eq. (2)), and the representative $C^{*}$ is unique up to a translation. We have the following

Proposition: A system $\mathfrak{S}_{i}$ evolving spontaneously tends to an asymptotic configuration $C^{t_{\infty}}$, and it is $C^{t_{\infty}} \in \mathfrak{C}^{*} \forall i \in \mathbb{N}: 1 \leq i \leq N$.

Proof: Let us first prove that the limit configuration exists. Then we will prove the Lemma by showing that, if $C^{t_{\infty}}$ is the asymptotic configuration, one has $\mathscr{E} D\left(C^{t_{\infty}}\right)=0$ (the thesis follows then immediately).

We start by noticing that in every virtual time step one element or two elements which are not neighbors move to the centroid of their first neighbors, while all other elements do not move. Since the centroid locally minimizes the discrete energy, this means that $\mathscr{E}^{D}\left(V_{h}, t_{j}\right) \geq \mathscr{E}^{D}\left(V_{h+1}, t_{j}\right)$ and $\mathscr{E}^{D}\left(V_{h}, t_{j}\right) \geq \mathscr{E}^{D}\left(V_{k}, t_{j+1}\right)$ for all integers $j$, and for all $h$ and $k$ for which virtual configurations are defined. Since actual configurations of the system are a subset (preserving the order relation) of the virtual configurations, one has that $\mathscr{E}^{D}\left(C^{t_{j}}\right)\left(t_{j} \in \mathfrak{T}\right)$ is a monotonically decreasing function of $j$. Since $\mathscr{E} D$ is non negative, this means that there exists $\lim _{m \rightarrow \infty} \mathscr{E}^{D}\left(C^{t_{m}}\right)=\mathscr{E}^{D}\left(C^{t_{\infty}}\right)$. Let us now enumerate all virtual configurations (including the ones identified by definition with actual ones): $V_{1}, V_{2}, \ldots V_{n}, \ldots$. Since $\mathscr{E} D$ converges, one has:

$$
\Delta \mathscr{E}^{D}\left(V_{h}\right):=\mathscr{E}^{D}\left(V_{h}\right)-\mathscr{E}^{D}\left(V_{h}+1\right) \rightarrow 0 \text { for } m \rightarrow \infty
$$

Let $\Delta s_{h}$ be the sum of the moduli of the displacements of the elements that move in the virtual time step $V_{h}$. Since $\Delta \mathscr{E}^{\mathscr{D}}\left(V_{h}\right)$ is a continuous function of $\Delta s_{h}$ vanishing if and only if $\Delta s_{h}=0$, Eq. (8) implies that $\lim _{h \rightarrow \infty} \Delta s_{h}=0$, i.e. all virtual displacements of the elements (and thus the actual ones) tend to zero as time goes to infinity. Therefore, there exists a limit configuration $C^{t_{\infty}}$.

Let us now suppose by contradiction that $\mathscr{E} D\left(C^{t_{\infty}}\right)=\tilde{\mathscr{E}}>0$ for some element $\bar{i}$ chosen as the pseudo-leader.

Let now $d_{\max }$ and $d_{\min }$ be respectively the maximum and minimum distance between (non fictitious) elements which are first neighbors in $C^{t_{\infty}}$. Since $\tilde{\mathscr{E}}>0$, one has that at least one between the two inequalities $d_{\max }>1$ and $d_{\min }<1$ holds; without losing generality, we select the first possibility. Let $\tilde{i}$ and $\tilde{i}+1$ be the two Lagrangian elements such that

$$
\lim _{m \rightarrow \infty}\left(\left|x_{\tilde{i}+1}^{t_{m}}-x_{\tilde{i}}^{t_{m}}\right|\right)=d_{\max }
$$

Recalling that the centroid algorithm moves the element $\tilde{i}$ to the centroid of its neighbors, it easily follows from Eq.(9) that: 


$$
\lim _{m \rightarrow \infty}\left(\left|x_{\tilde{i}}^{t_{m}}-x_{\tilde{i}-1}^{t_{m}}\right|\right)=d_{\max }
$$

Let $x_{F}^{t_{m}}$ be the coordinate of the left fictitious element of the system at time $t_{m}$. Iterating the previous reasoning, one gets eventually

$$
\lim _{m \rightarrow \infty}\left|x_{0}^{t_{m}}-x_{F}^{t_{m}}\right|=d_{\max }>1
$$

and since by definition $\left|x_{0}-x_{F}\right|=1$ at every time, the contradiction completes the proof.

Remark The result is easily generalized to system with second or higher order interaction. As mentioned in the paper, however, the generalization to 2D systems is not straightforward as in that case it is not true that the energy is decreasing.

\section{References}

1. G. A. Francfort and J.-J. Marigo, Journal of the Mechanics and Physics of Solids 46, 1319 (1998).

2. B. Bourdin, G. A. Francfort, and J.-J. Marigo, Journal of elasticity 91, 5 (2008).

3. W. Ching, P. Rulis, and A. Misra, Acta Biomaterialia 5, 3067 (2009).

4. M. Kulkarni, S. Pal, and D. Kubair, International journal of solids and structures 44, 229 (2007).

5. A. Rinaldi and L. Placidi, ZAMM-Journal of Applied Mathematics and Mechanics/Zeitschrift für Angewandte Mathematik und Mechanik 94, 862 (2014).

6. A. Della Corte, A. Battista, et al., International Journal of Non-Linear Mechanics 80, 209 (2016).

7. A. Battista, L. Rosa, R. dell'Erba, and L. Greco, Mathematics and Mechanics of Solids doi: 10.1177/1081286516657889 (2016).

8. R. D. Mindlin, International Journal of Solids and Structures 1, 417 (1965).

9. F. dell'Isola and P. Seppecher, Meccanica 32, 33 (1997).

10. F. dell'Isola, G. Sciarra, and S. Vidoli, Proceedings of the Royal Society of London A: Mathematical, Physical and Engineering Sciences doi: 10.1098/rspa.2008.0530 (2009).

11. L. Placidi, Continuum Mechanics and Thermodynamics 27, 623 (2015).

12. R. Sunyk and P. Steinmann, International Journal of Solids and Structures 40, 6877 (2003).

13. A. C. Eringen, Microcontinuum field theories: I. Foundations and Solids (Springer Science \& Business Media, 2012).

14. P. Germain, SIAM Journal on Applied Mathematics 25, 556 (1973).

15. R. D. Mindlin, Archive for Rational Mechanics and Analysis 16, 51 (1964).

16. P. Steinmann, International Journal of Solids and Structures 31, 1063 (1994).

17. J. Altenbach, H. Altenbach, and V. A. Eremeyev, Archive of Applied Mechanics 80, 73 (2010).

18. L. Placidi, S. H. Faria, and K. Hutter, Annals of Glaciology 39, 49 (2004).

19. V. Eremeyev, E. Ivanova, N. Morozov, and S. Strochkov, in Doklady Physics, Vol. 52 (Springer, 2007) pp. 699-702.

20. A. Madeo, L. Placidi, and G. Rosi, Research in Nondestructive Evaluation 25, 99 (2014).

21. V. Eremeyev, in Doklady Physics, Vol. 50 (Springer, 2005) pp. 204-206.

22. C. S. Chang and A. Misra, Journal of engineering mechanics 116, 2310 (1990).

23. F. Dell'Isola, D. Steigmann, and A. Della Corte, Applied Mechanics Reviews 67, 21 (2016).

24. G. Thiagarajan and A. Misra, International Journal of Solids and Structures 41, 2919 (2004).

25. S. J. A. Koh, H. P. Lee, C. Lu, and Q. H. Cheng, Physical Review B 72, 085414 (2005). 
26. A. Misra, L. A. Roberts, and S. M. Levorson, Geotechnical and Geological Engineering 25, 65 (2007).

27. S.-P. Kim, A. C. Van Duin, and V. B. Shenoy, Journal of Power Sources 196, 8590 (2011).

28. M. E. Tuckerman, Journal of Physics: Condensed Matter 14, R1297 (2002).

29. M. C. Payne, M. P. Teter, D. C. Allan, T. Arias, and J. Joannopoulos, Reviews of Modern Physics 64, 1045 (1992).

30. M. P. Allen, Computational Soft Matter: From Synthetic Polymers to Proteins 23, 1 (2004).

31. E. S. Levitan, the Journal of the Acoustical Society of America 32, 1265 (1960).

32. M. Kot, H. Nagahashi, and P. Szymczak, The Visual Computer 31, 1339 (2015).

33. R. E. D. Bishop and D. C. Johnson, The mechanics of vibration (Cambridge University Press, 2011).

34. T. J. Hughes, J. A. Cottrell, and Y. Bazilevs, Computer methods in applied mechanics and engineering 194, 4135 (2005).

35. W. A. Wall, M. A. Frenzel, and C. Cyron, Computer methods in applied mechanics and engineering 197, 2976 (2008).

36. L. Greco and M. Cuomo, Computer Methods in Applied Mechanics and Engineering 269, 173 (2014).

37. A. Cazzani, M. Malagù, and E. Turco, Continuum Mechanics and thermodynamics 28, 139 (2016).

38. Y. Toklu, Computers \& structures 82, 1581 (2004).

39. R. Temür, Y. S. Türkan, and Y. C. Toklu, in Recent Advances in Swarm Intelligence and Evolutionary Computation (Springer, 2015) pp. 283-300.

40. A. Kaveh and S. Talatahari, in Harmony search algorithms for structural design optimization (Springer, 2009) pp. 159-198.

41. M. Clerc, Particle swarm optimization, Vol. 93 (John Wiley \& Sons, 2010).

42. M. Vaz Jr, E. Cardoso, and J. Stahlschmidt, Engineering Computations 30, 936 (2013).

43. M. Ballerini, N. Cabibbo, R. Candelier, A. Cavagna, E. Cisbani, I. Giardina, V. Lecomte, A. Orlandi, G. Parisi, A. Procaccini, et al., Proceedings of the national academy of sciences 105, 1232 (2008).

44. N. Bellomo and J. Soler, Mathematical Models and Methods in Applied Sciences 22, 1140006 (2012).

45. N. Bellomo and C. Dogbe, Mathematical Models and Methods in Applied Sciences 18, 1317 (2008).

46. N. Bellomo, D. Knopoff, and J. Soler, Mathematical Models and Methods in Applied Sciences 23, 1861 (2013).

47. S. Berrimi and S. A. Messaoudi, Electronic journal of differential equations 2004, 1 (2004).

48. S. Berrimi and S. A. Messaoudi, Nonlinear Analysis: Theory, Methods \& Applications 64, 2314 (2006).

49. S. A. Messaoudi and N.-e. Tatar, Nonlinear Analysis: Theory, Methods \& Applications 68, 785 (2008).

50. F. Liang and H. Gao, Boundary Value Problems 2011, 1 (2011).

51. G. C. Sih, Mechanics of fracture initiation and propagation: surface and volume energy density applied as failure criterion, Vol. 11 (Springer Science \& Business Media, 2012).

52. A. Luongo, Thin-Walled Structures 48, 744 (2010).

53. A. Luongo, Mathematics and Mechanics of Solids , doi: 1081286515616053 (2015).

54. A. Luongo, Journal of sound and vibration 155, 249 (1992).

55. G. Piccardo, L. C. Pagnini, and F. Tubino, Continuum Mechanics and Thermodynamics 27, 261 (2015).

56. N. L. Rizzi, V. Varano, and S. Gabriele, Thin-Walled Structures 68, 124 (2013). 